\title{
The efficacy and safety of selective COX-2 inhibitors for postoperative pain management in patients after total knee/ hip arthroplasty: a meta-analysis
}

\author{
Mingyang Jiang ${ }^{1}$, Huachu Deng ${ }^{2}$, Xuxu Chen², Yunni Lin², Xiaoyong Xie ${ }^{2}$ and Zhandong Bo ${ }^{1 *}$ (D)
}

\begin{abstract}
Background: Many selective cyclooxygenase (COX-2) inhibitors are currently used in clinical practice. COX-2 inhibitors have good anti-inflammatory, analgesic, antipyretic effects, and gastrointestinal safety. However, the analgesic effects and adverse reactions of COX-2 after total knee/hip arthroplasty (TKA/THA) are not fully known.

Objective: To evaluate the efficacy and safety of selective COX-2 inhibitors in postoperative pain management in patients receiving TKA/THA.

Methods: Randomized controlled trials (RCTs) were retrieved from medical literature databases. Risk ratios (RR) Std mean difference (SMD) and 95\% confidence intervals (CI) were calculated to analyze the primary and safety endpoints.

Results: In total, 18 articles (23 trial comparisons) were retrieved comprising 3104 patients. Among them, 1910 patients (61.5\%) were randomized to the experimental group whereas 1194 patients (38.5\%) were randomized to the control group. The primary endpoints were the patients' VAS score at rest or on ambulation (within 3 days). We found that VAS score in patients that received selective COX-2 inhibitor was significantly lower compared to those of the control group.
\end{abstract}

Conclusion: This meta-analysis shows that selective COX-2 inhibitor therapy is effective, safe, and reliable in relieving postoperative pain of THA/TKA.

Keywords: Selective COX-2 inhibitor, Total knee arthroplasty, Total hip arthroplasty, Meta-analysis

\section{Introduction}

Total hip or knee arthroplasty (THA/TKA) is commonly performed to alleviate the symptoms of hip or knee joint dysfunction, promote the recovery of the disease, and improve the quality of life of patients [1]. However, postoperative pain has been a major drawback as it directly affects postoperative rehabilitation of patients [2].

Currently, non-steroidal anti-inflammatory drugs (NSAIDs) are often used for postoperative analgesia [3]. Traditional NSAIDs (such as fotaline, ibuprofen) achieve exert analgesic effects by non-selectively inhibiting cyclooxygenase

\footnotetext{
* Correspondence: drrbozhandong@126.com

'Department of Bone and Joint Surgery, The First Affiliated Hospital of

Guangxi Medical University, Nanning, Guangxi, China

Full list of author information is available at the end of the article
}

(COX) [4]. COX comprises two isozyme isomers, COX-1 and COX-2. COX-1 is an inherent housekeeping enzyme [5], mainly distributed in the stomach, kidney, and platelets. It catalyzes the production of physiologically needed prostaglandin E2 (PGE2) that regulates peripheral vascular resistance, platelet aggregation, maintains renal blood flow, and protects gastric mucosa [6]. On the other hand, COX-2 is expressed by monocytes, macrophages, fibroblasts etc., in response to inflammatory stimulation, and thus, it is referred to as inducible enzyme [7]. It is one of the key enzymes that initiate inflammatory reactions and promote inflammatory response leading to tissue injury [8]. NSAIDs, therefore, simultaneously exert anti-inflammation and analgesic effects which also increases the risk of perioperative 
bleeding and digestive tract symptoms [9]. Selective COX2 inhibitors not only prevent inflammation and exert analgesic and antipyretic effects, but also protect the gastrointestinal mucosa and are widely used in orthopedic postoperative analgesia [10].

Although COX-2 inhibitors can relieve postoperative pain, their analgesic and adverse effects have not been fully analyzed [11]. This meta-analysis was conducted to explore the efficacy and safety of COX-2 inhibitors in postoperative pain management for patients receiving THA/TKA to provide reference data for clinical guidance.

\section{Methods}

\section{Search strategy}

Two researchers searched for published articles analyzing the efficacy and safety of selective COX-2 inhibitors in postoperative pain management for patients undergoing THA/TKA. We then performed a meta-analysis following the Preferred Reporting Items for Systematic Reviews and Meta-Analyses (PRISMA) guidelines. The randomized controlled trials (RCTs) were systematically searched in databases including PubMed, Embase, the Cochrane Library, Baidu Scholar, Google Scholar, CNKI, and VIP with no restrictions on language or publication date from inception to 12 May 2019. Additional relevant studies were retrieved from reviews, meta-analyses, and other literature. Two authors screened and doublereviewed the retrieved studies. In cases of discrepancies, a third researcher was consulted to obtain a consensus. In this meta-analysis, all data were extracted from previously published studies; thus, patient consent and ethical approval were not required.

\section{Inclusion and exclusion criteria}

We included clinical trials analyzing the efficacy and safety of selective COX-2 inhibitors in patients with THA or TKA and RCTs involving selective COX-2 inhibitors, in which, all patients underwent TKA or THA. The following types of studies were excluded: retrospective trials, animal experiments, non-randomized clinical trials, reviews, series, and case reports; studies with erroneous or incomplete data; studies that did not focus on TKA or THA patients; and studies in which patients were allergic to selective COX-2 inhibitors.

\section{Endpoints}

In this meta-analysis, the primary endpoint was the VAS score within 3 days after surgery. The secondary endpoint was morphine supplementation within 3 days after surgery. The safety endpoints included nausea, vomiting, pruritus, dizziness, fever, edema, lethargy, insomnia, constipation, diarrhea, and headache.

\section{Data extraction}

Two authors independently reviewed the contents of the retrieved studies. The primary endpoints were extracted by two authors and verified by a third author. The data extracted included the following primary information: first author's name, year of publication, test type/region, sample size, sex ratio, average age, intervention, supplemental analgesic drugs, type of surgery, follow-up time, and endpoints measured in each study. If the content of the studies needed clarification, the first author of the study was contacted. Disagreements were resolved through consensus or by consulting a third author.

\section{Risk-of-bias assessments}

The methodological quality of the included studies was estimated independently by two authors based on the Cochrane Risk of Bias criteria. Seven items were used to assess bias in each trial, i.e., randomization sequence generation, allocation concealment, blinding of participants and personnel, blinding of outcome assessment, incomplete outcome data, selective reporting, and other biases. Each quality item was graded as low risk, high risk, or no clear risk.

\section{Statistical analysis}

Stata (version 12.0, Stata Corp, College Station, Texas) was used to analyze and pool the individual research findings. Pooled results were recorded as risk ratios (RR), Std mean difference (SMD), and 95\% confidence intervals $(\mathrm{CI})$ with two-sided $p$ values. $p$ values $<0.05$ were considered to be statistically significant. Heterogeneity was evaluated using the $I^{2}$ test. The heterogeneity was considered to be small when $I^{2}<50 \%$ and substantial when $I^{2}>50 \%$. The fixed effect model was used when $I^{2}<50 \%$, while the random effect model was used when $I^{2}>50 \%$. A funnel plot was generated to examine the publication bias and to explore the sources of heterogeneity if more than ten studies were included to assess this endpoint. Subgroup analysis was performed according to the administration and type of selective COX-2 inhibitor.

\section{Results}

\section{Characteristics of retrieved studies}

In total, 1428 relevant studies were identified in line with the PRISMA guidelines. The titles and abstracts of the studies were screened to exclude irrelevant studies. After reading the full text of the identified studies, we excluded those that did not meet the set criteria. Finally, 17 studies (22 trial comparisons) comprising 2919 patients were enrolled in this meta-analysis as shown in Fig. 1. Among the 2919, 1819 patients (62.3\%) were randomized to the selective $\mathrm{COX}-2$ inhibitor group whereas 1100 patients $(37.7 \%)$ were randomized to the 


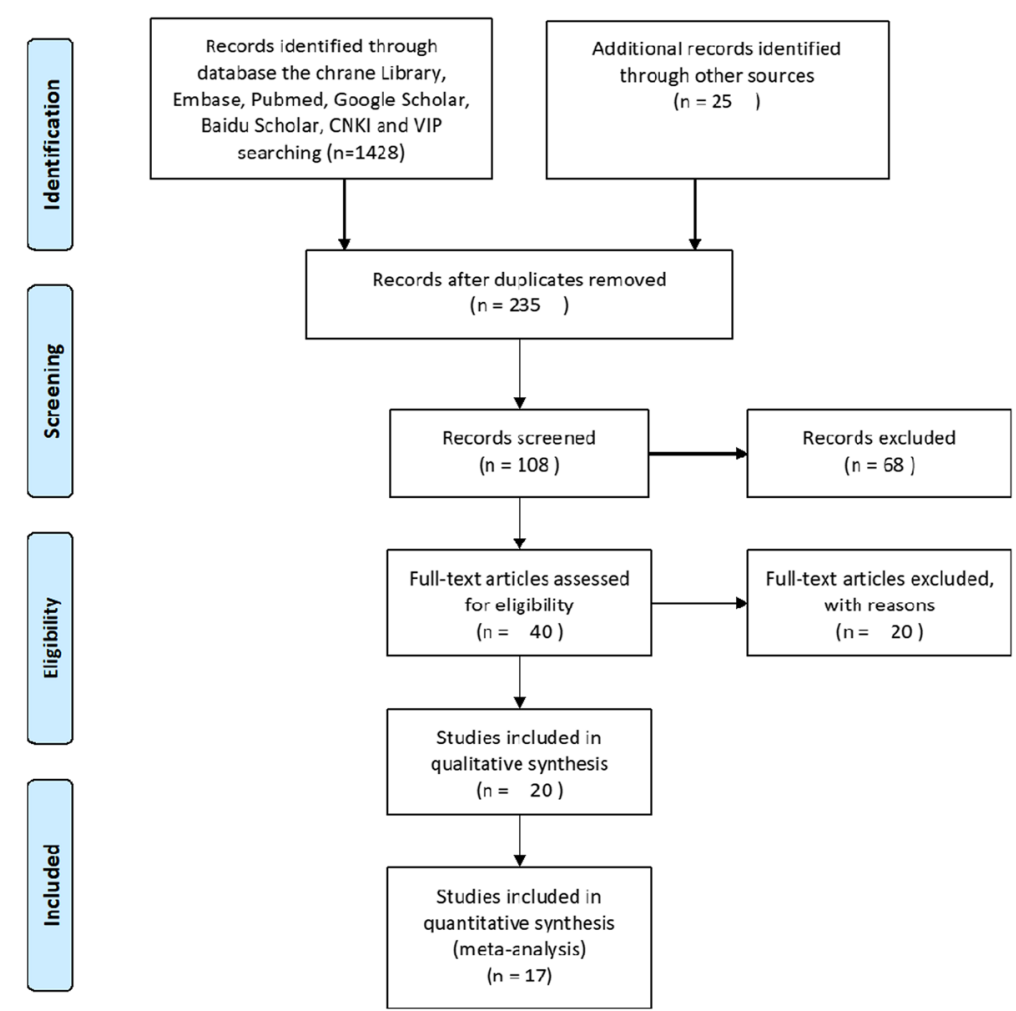

Fig. 1 Flow diagram of the study selection process. CNKI, China national knowledge infrastructure; VIP, China Science and Technology Journal Database

control group. All studies included in this meta-analysis were RCTs. The basic characteristics of the participants in each trial are shown in Table 1.

\section{Evaluating literature quality}

The Cochrane Risk of Bias criteria was used to evaluate the quality of the enrolled studies, which was conducted by two researchers. All included studies were randomized controlled trials. In summary, 17 studies [12-28] described random sequence generation and allocation concealment. Nine studies [12-14, 20, 22, 24, 25, 27, 28] described the blinding of participants and personnel. Nine studies [12-14, 20, 22, 24, 25, 27, 28] described the blinding of outcome assessment. None of the studies described other biases. The literature quality scores of each study are shown in Table 2. The random effect model was applied to determine the level of heterogeneity among the articles.

\section{Primary endpoints}

\section{VAS score at rest within 3 days post-surgery}

Five studies [16, 18, 21, 26, 27] (5 trial comparisons) reported VAS score at rest within 3 days after surgery. In total, VAS score at rest was provided for 275 patients, of whom 138 were assigned to the selective COX-2 inhibitor group and 137 were assigned to the control group.
The result showed that the VAS scores at rest in the selective COX-2 inhibitor group were significantly lower compared to the control group (SMD - 1.22, 95\% CI 1.72 to $-0.73, I^{2}=89.4 \%$ ) as shown in Fig. 2. The random effect model was applied to determine the level of heterogeneity among the articles. The subgroup analysis revealed that the VAS scores at rest in selective COX-2 inhibitor group were significantly lower than that in the control group at 24 and $48 \mathrm{~h}$ (SMD - 1.17, 95\% CI 1.96 to -0.37 ; SMD $-1.15,95 \% \mathrm{CI}-1.92$ to -0.38 ). However, the VAS scores were not significantly different between the selective COX-2 inhibitor and control groups at $72 \mathrm{~h}$ (SMD $-1.47,95 \% \mathrm{CI}-3.02$ to -0.08$)$.

\section{VAS scores after ambulation within 3 days post-surgery}

Seven studies [16-19, 23, 26, 27] (8 trial comparisons) reported VAS scores for ambulation within 3 days after surgery. In total, VAS scores after ambulation were reported for 482 patients, of which 274 were assigned to the selective COX-2 inhibitor group and 208 were assigned to the control group. It was observed that VAS scores after ambulation in the selective COX-2 inhibitor group were significantly lower than that of the control group (SMD $1.23,95 \% \mathrm{CI}-1.54$ to $-0.93, I^{2}=84.9 \%$ ) as shown in Fig. 3. The random effect model was applied to determine the level of heterogeneity among the articles. The 


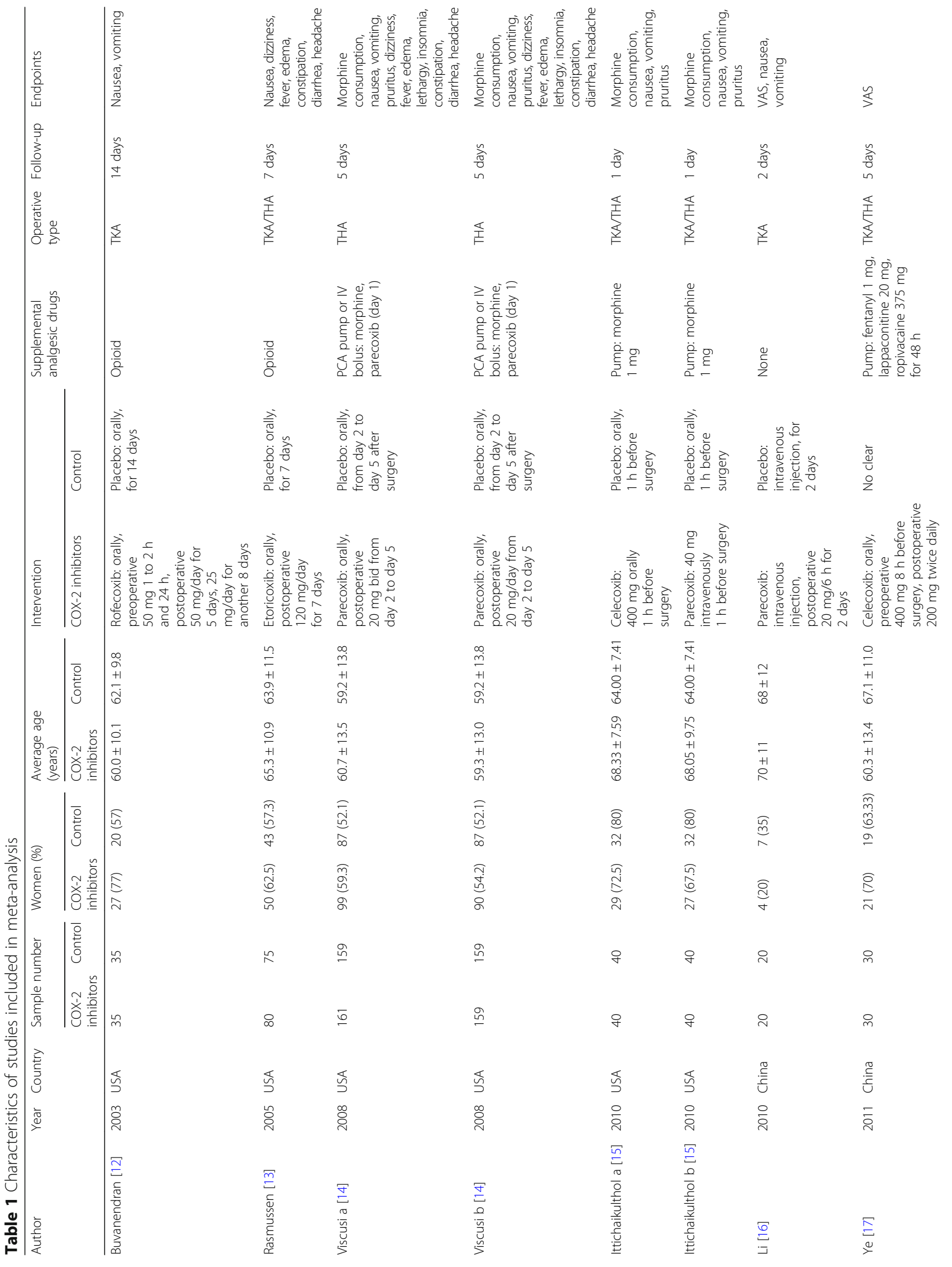




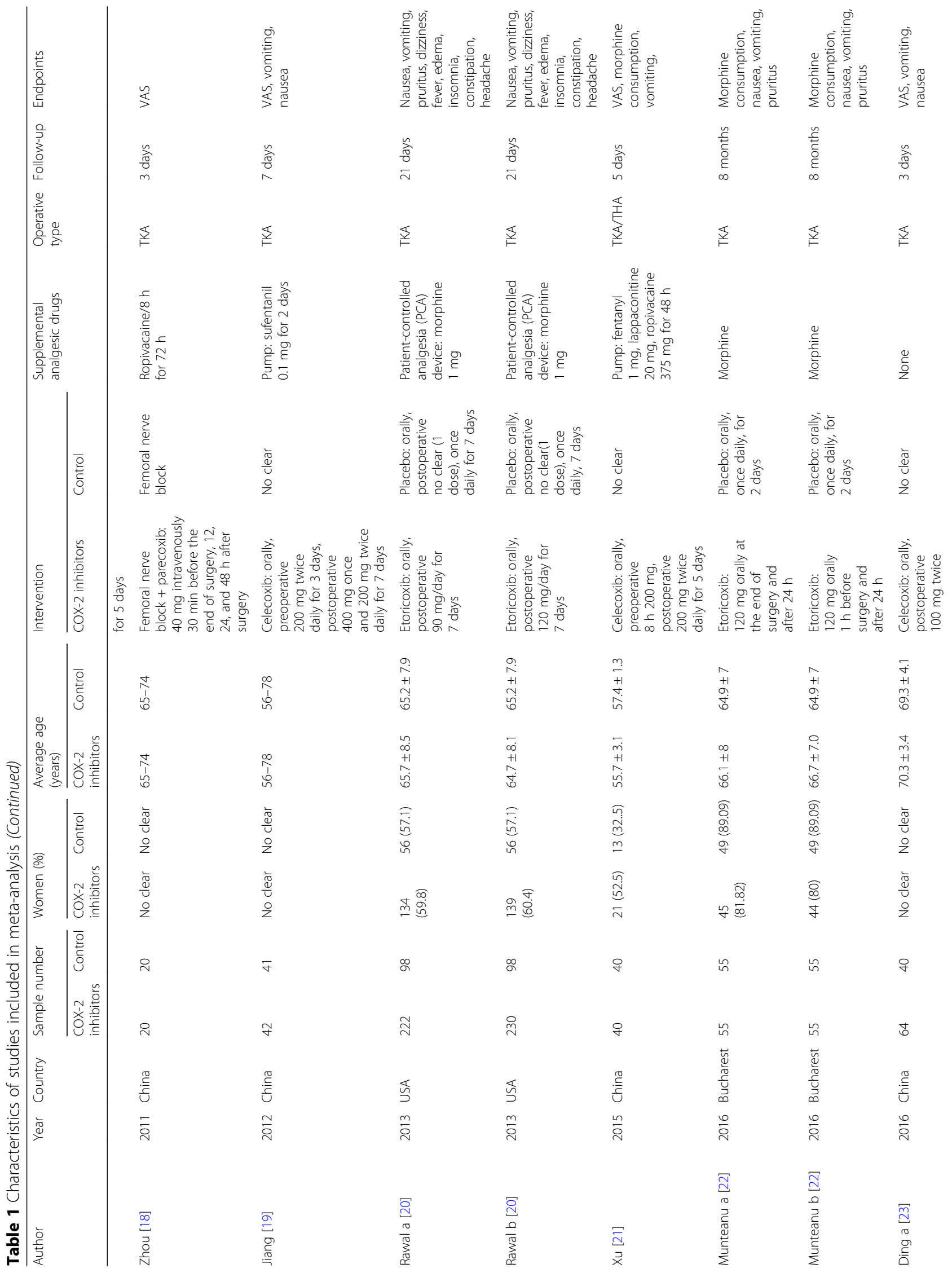




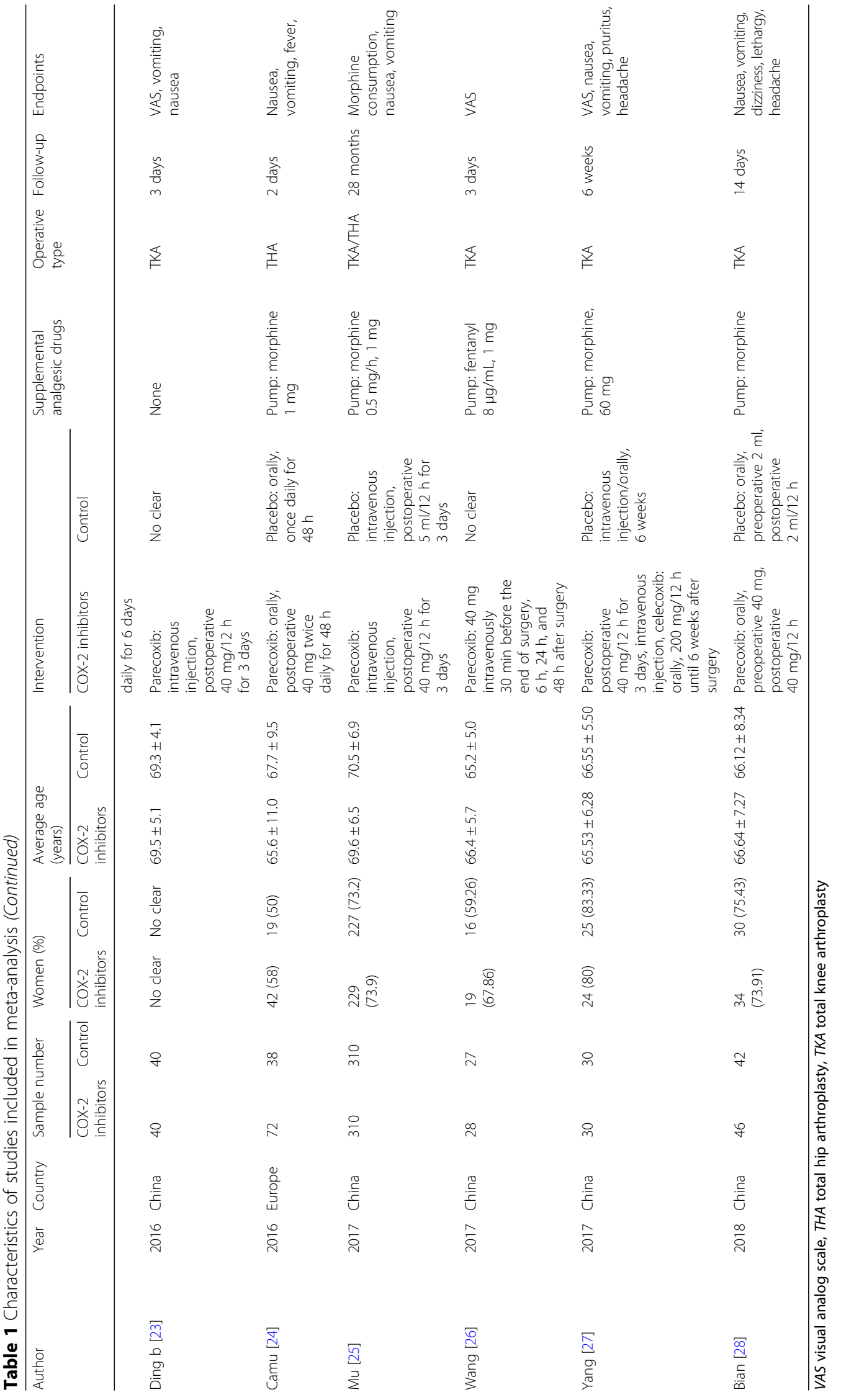


Table 2 Assessment of methodological quality of included studies

\begin{tabular}{|c|c|c|c|c|c|c|c|}
\hline Study & Random allocation & Hidden distribution & Blind method & $\begin{array}{l}\text { Incomplete } \\
\text { outcome data }\end{array}$ & $\begin{array}{l}\text { Selective reporting } \\
\text { of results }\end{array}$ & Other bias & Quality grade \\
\hline Buvanendran [12] & Randomized & No clear & Double-blind & Low & Low & Low & C \\
\hline Rasmussen [13] & Randomized & No clear & Double-blind & Low & Low & Low & B \\
\hline Viscusi a [14] & Randomized & No clear & Double-blind & Low & Low & Low & B \\
\hline Viscusi b [14] & Randomized & No clear & Double-blind & Low & Low & Low & B \\
\hline Ittichaikulthol a [15] & Randomized & No clear & No clear & Low & Low & Low & C \\
\hline Ittichaikulthol b [15] & Randomized & No clear & No clear & Low & Low & Low & C \\
\hline Li [16] & Randomized & No clear & No clear & Low & Low & Low & C \\
\hline Ye [17] & Randomized & No clear & No clear & Low & Low & Low & C \\
\hline Zhou [18] & Randomized & No clear & No clear & Low & Low & Low & C \\
\hline Jiang [19] & Randomized & No clear & No clear & Low & Low & Low & C \\
\hline Rawal a [20] & Randomized & No clear & Double-blind & Low & Low & Low & B \\
\hline Rawal b [20] & Randomized & No clear & Double-blind & Low & Low & Low & B \\
\hline Xu [21] & Randomized & No clear & No clear & Low & Low & Low & C \\
\hline Munteanu a [22] & Randomized & No clear & Double-blind & Low & Low & Low & A \\
\hline Munteanu b [22] & Randomized & No clear & Double-blind & Low & Low & Low & A \\
\hline Ding a [23] & Randomized & No clear & No clear & Low & Low & Low & C \\
\hline Ding b [23] & Randomized & No clear & No clear & Low & Low & Low & C \\
\hline Camu [24] & Randomized & No clear & Double-blind & Low & Low & Low & A \\
\hline Mu [25] & Randomized & No clear & Double-blind & Low & Low & Low & A \\
\hline Wang [26] & Randomized & No clear & No clear & Low & Low & Low & C \\
\hline Yang [27] & Randomized & No clear & Double-blind & Low & Low & Low & C \\
\hline Bian [28] & Randomized & No clear & Double-blind & Low & Low & Low & B \\
\hline
\end{tabular}

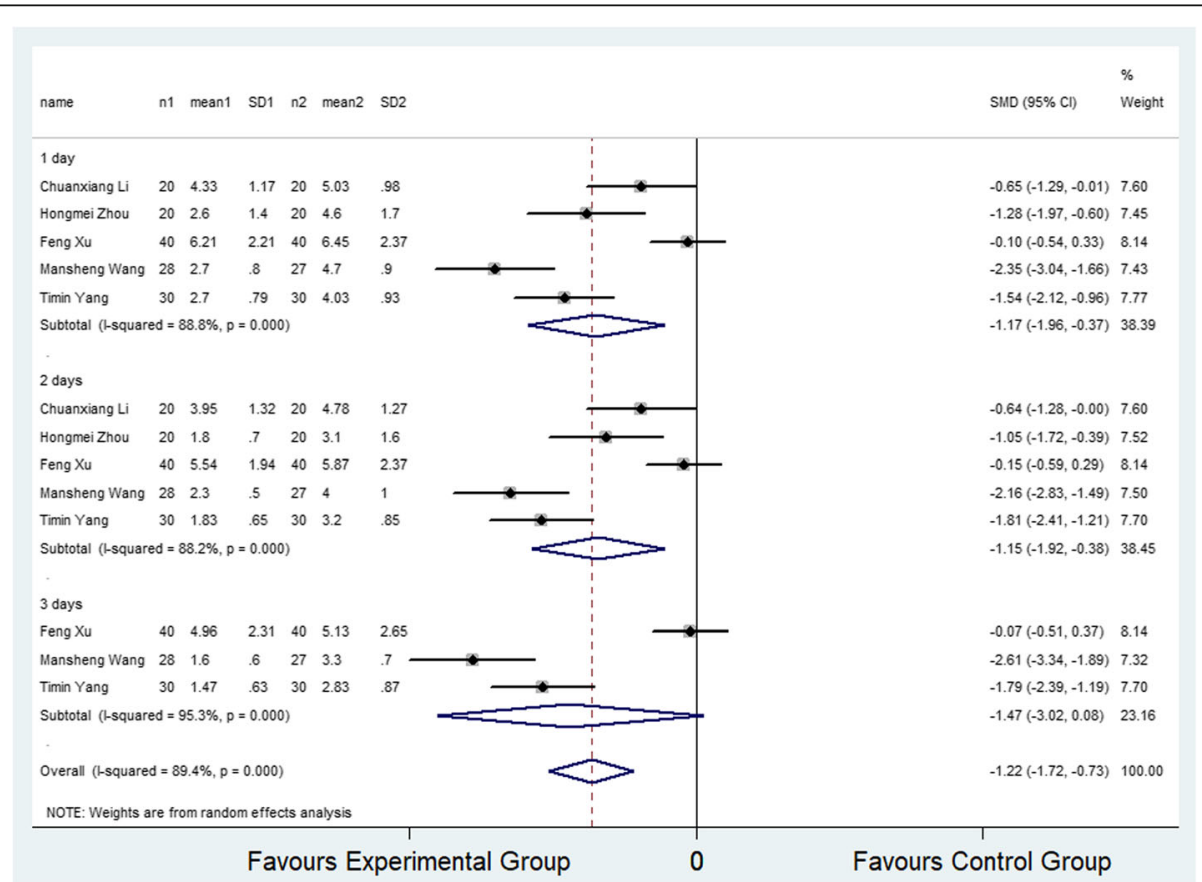

Fig. 2 Comparison of VAS score at rest within 3 days after surgery between the selective COX-2 inhibitor group and the control group. SMD, standardized mean difference 


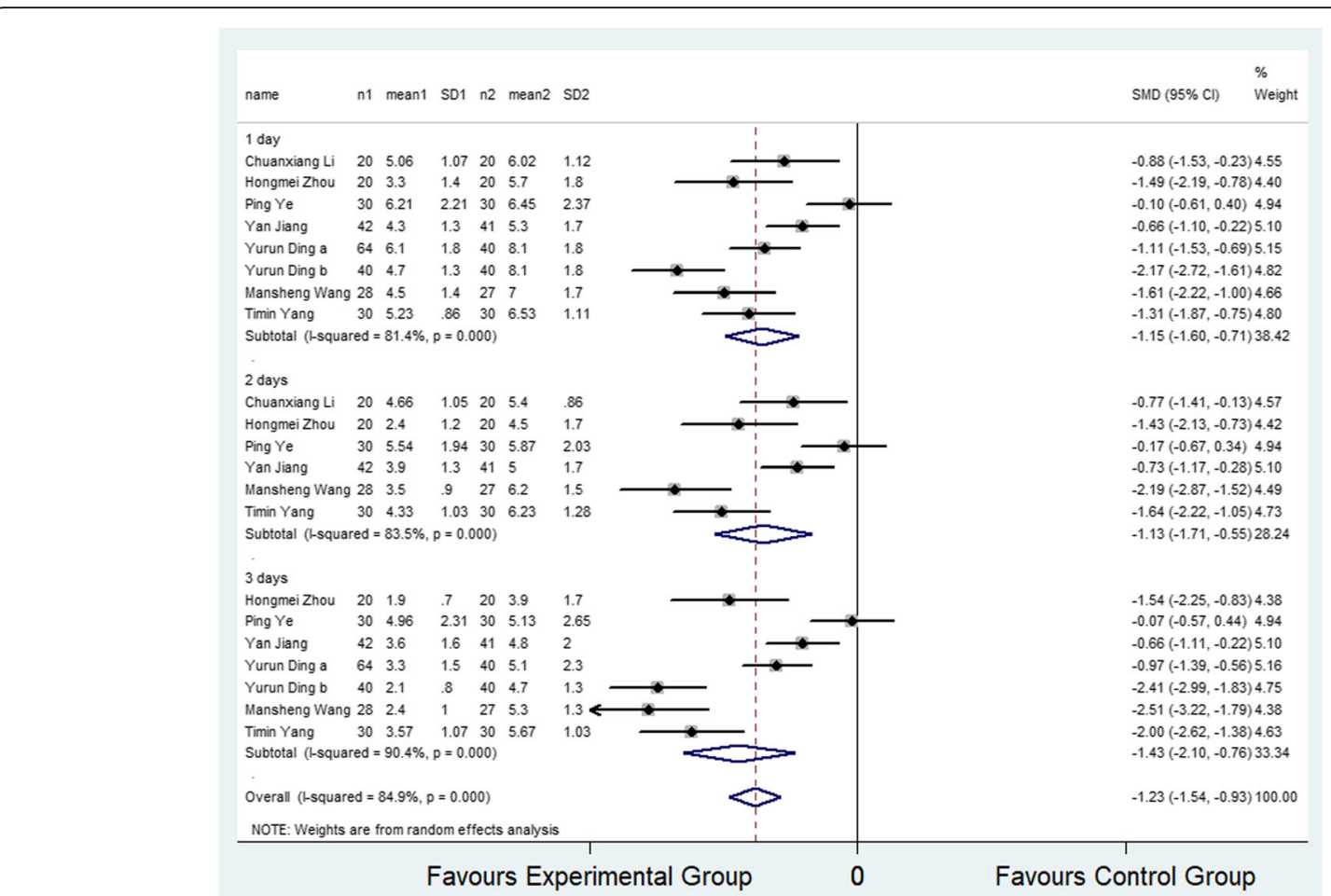

Fig. 3 Comparison of VAS score on ambulation within 3 days after surgery between the selective COX-2 inhibitor group and the control group. SMD, standardized mean difference

subgroup analysis revealed that VAS scores after ambulation in selective COX-2 inhibitor group were significantly lower than that of the control group at 24,48 , and $72 \mathrm{~h}$ (SMD - 1.15, 95\% CI - 1.60 to - 0.71; SMD - 1.13, 95\% CI -1.71 to -0.55 ; SMD $-1.43,95 \%$ CI -2.10 to -0.76 ).

In the administration subgroup analysis, it was found that the VAS scores in selective COX-2 inhibitor group were significantly lower than that of the control group when selective COX-2 inhibitors were injected (SMD - 1.55, 95\% CI -2.09 to -1.01 ; SMD $-1.46,95 \%$ CI -2.28 to -0.64 ; SMD $-2.17,95 \% \mathrm{CI}-2.74$ to -1.59 ) within 24 and $72 \mathrm{~h}$. Similar results were obtained when selective COX-2 inhibitors were orally administered (SMD $-0.64,95 \% \mathrm{CI}-1.20$ to -0.08 ; SMD $-0.59,95 \% \mathrm{CI}-1.09$ to -0.08$)$. There was no significant difference when selective COX-2 inhibitors were orally administered (SMD $-0.46,95 \% \mathrm{CI}-1.01$ to 0.09 ).

In the medication type subgroup analysis, it was observed that the VAS scores in the selective COX-2 inhibitor group were significantly lower than that of the control group when parecoxib (SMD - 1.55, 95\% CI 2.09 to -1.01 ; SMD $-1.46,95 \% \mathrm{CI}-2.28$ to -0.64 ; SMD $-2.17,95 \% \mathrm{CI}-2.74$ to -1.59 ) was administered within 24 and $72 \mathrm{~h}$. Similar results were obtained when celecoxib was administered (SMD $-0.64,95 \% \mathrm{CI}-1.20$ to 0.08 ; SMD $-0.59,95 \% \mathrm{CI}-1.09$ to -0.08$)$. There was no significant difference when using celecoxib (SMD -0.46 , $95 \% \mathrm{CI}-1.01$ to 0.09 ).

\section{Secondary endpoints}

\section{Morphine supplementation within 3 days after surgery}

Four studies [14, 15, 22, 25] (7 trial comparisons) reported morphine supplementation within 3 days after surgery. In total, 1383 patients received morphine supplementation, among whom 820 were assigned to the selective COX-2 inhibitor group and 563 were assigned to the control group. Figure 4 shows that the quantity of morphine supplementation in the selective COX-2 inhibitor group was significantly lower than that of the control group (SMD $-0.86,95 \% \mathrm{CI}-1.17$ to $\left.-0.56, I^{2}=94.7 \%\right)$. The random effect model was applied to determine the level of heterogeneity among the articles. In subgroup analysis, the quantity of morphine supplementation in the selective COX-2 inhibitor group was significantly lower than that of the control group at 24 and $48 \mathrm{~h}$ (SMD - 1.70, 95\% CI -2.59 to $-0.81, I^{2}=96.3 \%$; SMD $-0.59,95 \% \mathrm{CI}-1.05$ to -0.13 , $\left.I^{2}=94.1 \%\right)$. Notably, the difference between the two groups in morphine supplementation at $72 \mathrm{~h}$ was not significant (SMD $-0.34,95 \% \mathrm{CI}-0.75$ to $0.06, I^{2}=91.8 \%$ ).

\section{Safety endpoints}

Further analysis revealed that administration of selective COX-2 inhibitors significantly reduced the incidence of nausea (14.3\% vs $17.5 \%$ ) (RR $0.72,95 \%$ CI 0.61 to 0.85 ), vomiting (8.6\% vs $12.8 \%$ ) (RR $0.68,95 \%$ CI 0.54 to 0.85 ), and fever (7.3\% vs $17.6 \%$ ) (RR $0.36,95 \%$ CI 0.27 to 0.47 ). 


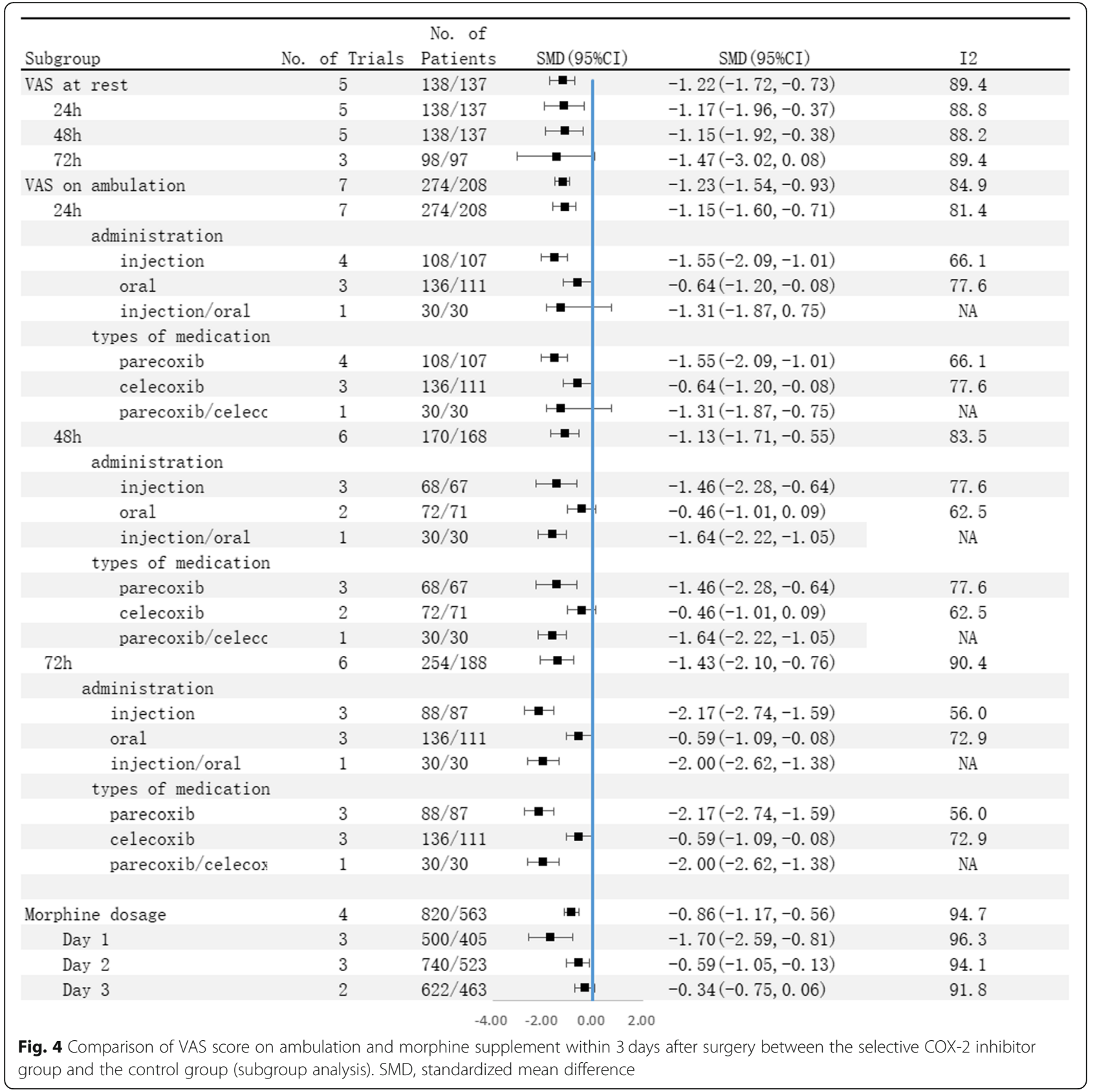

Moreover, there was no significant difference between the selective COX-2 inhibitor group and the control group in the occurrence of pruritus $(4.5 \%$ vs $4.7 \%$ ) (RR $0.85,95 \%$ CI 0.53 to 1.36 ), dizziness (5.3\% vs $5.8 \%$ ) (RR $0.90,95 \%$ CI 0.59 to 1.37 ), edema ( $2.8 \%$ vs $3.9 \%$ ) (RR $0.92,95 \%$ CI 0.53 to 1.59 ), lethargy (3.3\% vs $5.5 \%$ ) (RR $0.57,95 \%$ CI 0.29 to 1.14 ), insomnia $(7.4 \%$ vs $9.3 \%$ ) (RR $0.70,95 \%$ CI 0.47 to 1.02 ), constipation ( $12.8 \%$ vs $14.2 \%$ ) (RR $0.93,95 \%$ CI 0.71 to 1.22 ), diarrhea (1.8\% vs $3.0 \%$ ) (RR $0.63,95 \% \mathrm{CI}$ 0.25 to 1.57 ), and headache (4.1\% vs $5.9 \%$ ) (RR 0.79 , 95\% CI 0.50 to 1.25 ).

\section{Publication bias and sensitivity analysis}

A funnel plot showed that there was bias among the enrolled studies as shown in Additional file 1: Figure S1-S3. The results of the sensitivity analysis are shown in Additional file 2: Figure S4-S11.

\section{Discussion}

Postoperative pain severely limits limb movement and functional exercise after THA/TKA, and this compromises the recovery of postoperative joint function [29]. Currently, many selective COX-2 inhibitors are used in clinical practice [30]. These drugs have good anti-inflammatory, 
analgesic, antipyretic effects without causing gastrointestinal mucosal damage [31]. So far, the analgesic effects and adverse reactions of selective COX-2 inhibitors after THA/ TKA are not fully understood.

In recent years, research on of the application of selective COX-2 inhibitors has received tremendous attention. A review conducted by Parvizi et al. [32] provided strong evidence in favor of using NSAIDs and selective COX-2 inhibitors as part of the multimodal treatment plan in pain management. A meta-analysis by Ji et al. [33] indicated that preoperative administration of COX-2 inhibitor effectively improves postoperative analgesia, reduces the consumption of morphine, and lessens the incidence of pruritus. Other meta-analyses $[34,35]$ comparing the efficacy and safety of selective COX-2 inhibitors versus non-selective COX-2 inhibitors in the prevention of $\mathrm{HO}$ after THA revealed that selective COX-2 inhibitors are as effective as non-selective COX-2 inhibitor in preventing Heme oxygenase $(\mathrm{HO})$ after THA. Of note, selective COX-2 inhibitors were associated with fewer gastrointestinal side effects than non-selective COX-2 inhibitor. A preliminary study by $\mathrm{Oh}$ et al. [36], the efficacy of a selective COX-2 inhibitor in early postoperative pain control, pain management satisfaction level, and incidence of systemic adverse effects in patients undergoing arthroscopic rotator cuff repair were evaluated. This study concluded that, although the selective COX-2 inhibitors have similar postoperative analgesic effects as other nonsteroidal anti-inflammatory drugs and opioids, they might negatively affect tendon-to-bone healing after surgical repair, and thus, they should not be used for postoperative analgesia. Vastel et al. [37] compared the efficacy of celecoxib versus ketoprofen. They reported that celecoxib and ketoprofen had equivalent efficacy in reducing peri-prosthetic ossifications. Gong et al. [38] conducted an RCT to evaluate the efficacy of muscle relaxants and celecoxib in early recovery after total knee arthroplasty (TKA). They found that coapplication of celecoxib and eperisone with a low dose of morphine provided good and safe pain control in patients undergoing TKA. They summarized the efficacy of selective COX-2 inhibitor in early postoperative pain control, satisfaction with pain management, and incidence of systemic adverse effects in patients undergoing arthroscopic rotator cuff repair. Despite these findings, whether selective COX-2 inhibitors are effective for clinical postoperative pain management after TKA/THA deserves further analysis.

Unlike previous studies which separately explored TKA or THA, our meta-analysis examined both conditions simultaneously. This is also the first meta-analysis to explore the efficacy and safety of selective COX-2 inhibitors for postoperative pain management after TKA/THA. The primary endpoints show that the VAS scores at rest or on ambulation (within 3 days) in patients receiving selective COX-2 inhibitor are significantly lower than that of control subjects (SMD - 1.22, 95\% CI - 1.72 to - 0.73; SMD - 0.86, 95\% CI -1.17 to -0.56 ). Analysis of secondary endpoints shows that patients receiving selective COX-2 inhibitor require a smaller amount of morphine supplementation within 3 days after surgery (SMD $-0.86,95 \% \mathrm{CI}-1.17$ to -0.56 ) compared to the control subjects. Analysis of safety endpoints show that patients receiving selective COX-2 inhibitor have lower incidence of nausea, vomiting, and fever (RR 0.72, 95\% CI 0.61 to 0.85 ; RR 0.68 , 95\% CI 0.54 to 0.85 ; RR 0.36 , $95 \%$ CI 0.27 to 0.47 ) compared to the control subjects. Notably, the occurrence of pruritus, dizziness, lethargy, edema, insomnia, constipation, diarrhea, and headache were not significantly different between patients in the selective COX-2 inhibitor group and control group.

Results of the primary endpoints were highly heterogeneous, and thus, we performed sensitivity analyses to identify the sources of heterogeneity. The results show that the heterogeneity did not come from a certain article. The subgroup analyses performed according to the type of selective COX-2 inhibitor and mode of administration did not identify the source of heterogeneity. We

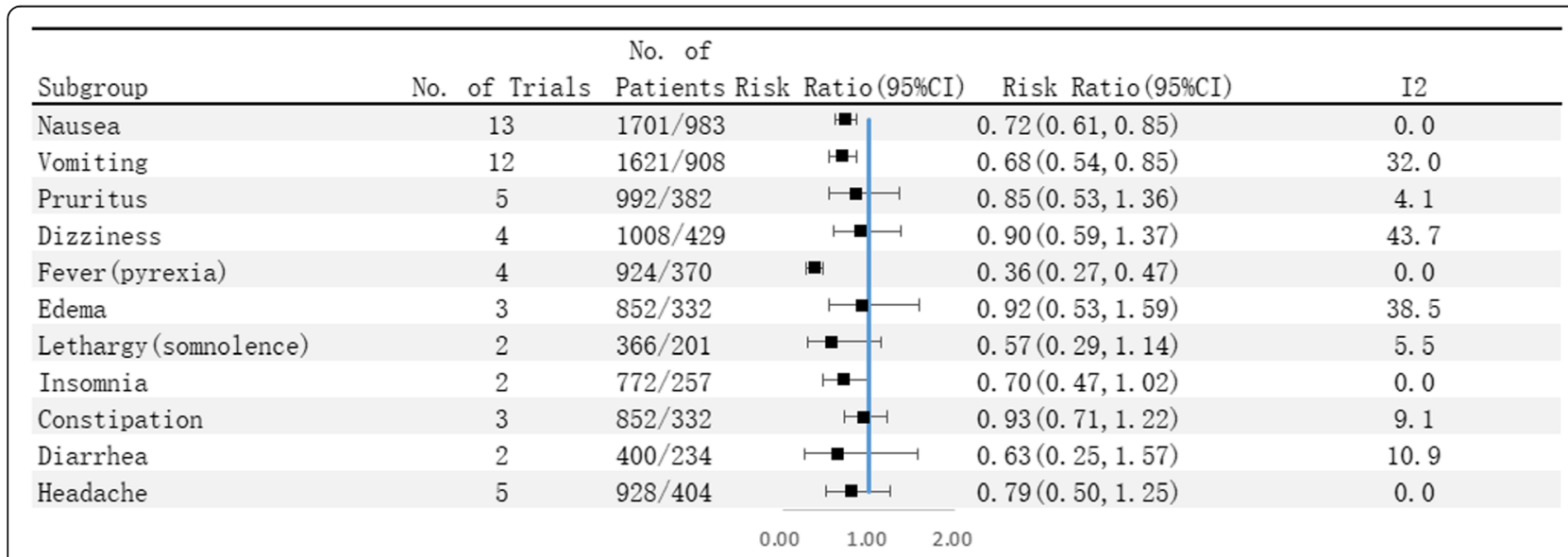

Fig. 5 Incidence of adverse reaction between the selective COX-2 inhibitor group and the control group. SMD, standardized mean difference 
thus postulate that heterogeneity among the studies may be caused by the small sample size in the studies.

The potential clinical implications of this meta-analysis are as follows: (1) This is the first study focusing on the efficacy and safety of selective COX-2 inhibitors for postoperative pain management after TKA/THA. A total of 17 RCTs were enrolled covering 2919 participants, which is a larger sample size compared to previous studies. (3) Sensitivity analyses and subgroup analyses were conducted to identify sources of heterogeneity and explore the influence of sample size on the overall effect. (4) We evaluated 13 indicators, including VAS score, morphine supplementation within 3 days after surgery, and the incidence of nausea, vomiting, fever, pruritus, dizziness, lethargy, edema, insomnia, constipation, diarrhea, and headache, making this meta-analysis more comprehensive than previous studies.

The limitations of this study are as follows: (1) Several baseline characteristics (diabetes, hypertension, older age, or other drug use) were not considered, and this may introduce mixed bias. (2) We used the outcome events reported in the identified studies. Therefore, it is difficult to assess the effect of these baseline characteristics on the results of this meta-analysis. (3) We did not explore the interactions among the subgroups because of the limitations inherent in the included studies. (4) The primary endpoints results were highly heterogeneous, and sensitivity analyses and subgroup analyses failed to identify the source of heterogeneity. (5) Because of the limited number of articles, the operative type and follow-up period subgroups were meaningless. (6) Only 9 studies described the blinding method used, pointing to the existence of mixed bias. (7) Many studies identified were small (with less than 1000 patients on average), and most of them were published in China and the USA. Thus, high-quality RCTs with large sample sizes are required to determine whether our results are applicable to other geographical regions. (8) Since most of the studies included provided interventions and supplementary analgesic drugs in the control group, the handling of labor pains is actually multimodal (although the authors of the included articles have controlled variables as much as possible). This may lead to high heterogeneity and ultimately affect the results. (9) The primary endpoints were analyzed at 24,48 , and $72 \mathrm{~h}$ after operation. However, given that the included studies did not mention the time point at which adverse reactions were recorded, subgroup analysis of secondary endpoints was not conducted according to time (Fig. 5).

This meta-analysis reveals that selective COX-2 inhibitors significantly reduce VAS scores at rest or on ambulation, and decreases morphine consumption within 3 days after surgery, the incidence of nausea, vomiting, and fever. This indicates that selective COX-2 inhibitors are highly effective and safe for postoperative pain control after TKA/THA.

\section{Supplementary information}

Supplementary information accompanies this paper at https://doi.org/10. 1186/s13018-020-1569-z.

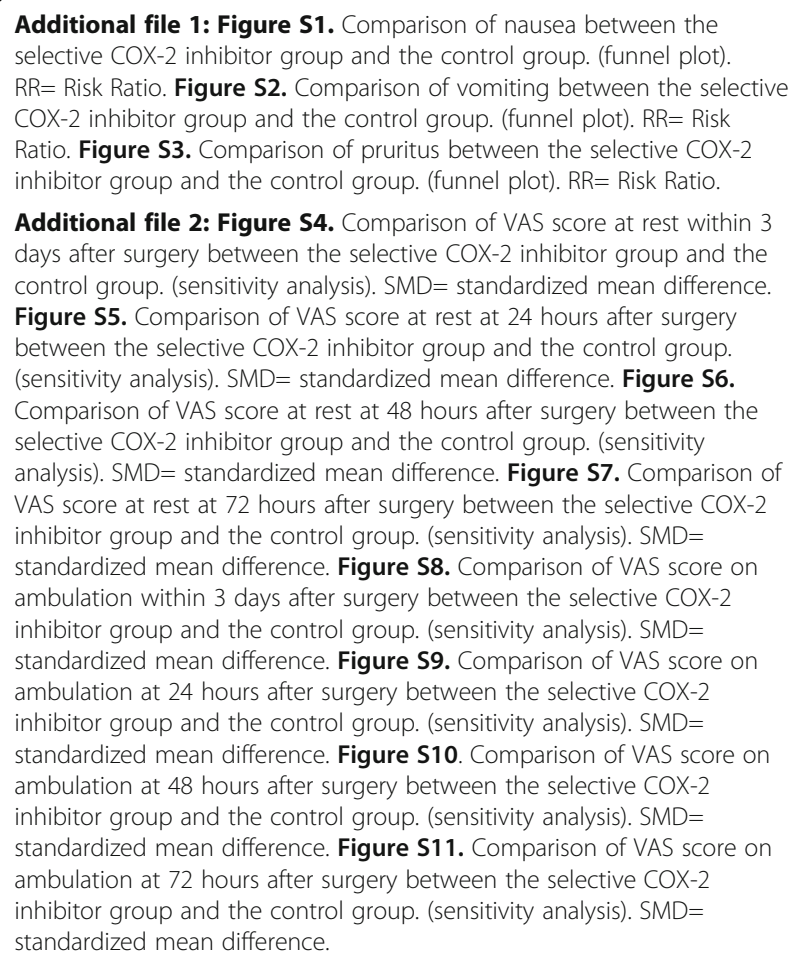

Additional file 1: Figure S1. Comparison of nausea between the selective COX-2 inhibitor group and the control group. (funnel plot). $\mathrm{RR}=$ Risk Ratio. Figure S2. Comparison of vomiting between the selective COX-2 inhibitor group and the control group. (funnel plot). RR= Risk Ratio. Figure S3. Comparison of pruritus between the selective COX-2 inhibitor group and the control group. (funnel plot). RR= Risk Ratio.

Additional file 2: Figure S4. Comparison of VAS score at rest within 3 days after surgery between the selective COX-2 inhibitor group and the control group. (sensitivity analysis). SMD= standardized mean difference. Figure S5. Comparison of VAS score at rest at 24 hours after surgery between the selective COX-2 inhibitor group and the control group. (sensitivity analysis). SMD= standardized mean difference. Figure S6. Comparison of VAS score at rest at 48 hours after surgery between the selective COX-2 inhibitor group and the control group. (sensitivity analysis). SMD= standardized mean difference. Figure S7. Comparison of VAS score at rest at 72 hours after surgery between the selective COX-2 inhibitor group and the control group. (sensitivity analysis). SMD= standardized mean difference. Figure S8. Comparison of VAS score on ambulation within 3 days after surgery between the selective COX-2 inhibitor group and the control group. (sensitivity analysis). SMD= standardized mean difference. Figure S9. Comparison of VAS score on ambulation at 24 hours after surgery between the selective COX-2 inhibitor group and the control group. (sensitivity analysis). SMD= standardized mean difference. Figure S10. Comparison of VAS score on ambulation at 48 hours after surgery between the selective COX-2 inhibitor group and the control group. (sensitivity analysis). SMD= standardized mean difference. Figure S11. Comparison of VAS score on ambulation at 72 hours after surgery between the selective COX-2 inhibitor group and the control group. (sensitivity analysis). $\mathrm{SMD}=$ standardized mean difference.

\section{Abbreviations}

Cl: Confidence intervals; COX: Cyclooxygenase; NSAIDs: Non-steroidal antiinflammatory drugs; PRISMA: Reporting Items for Systematic Reviews and Meta-Analyses; RCTs: Randomized controlled trials; RR: Risk ratios; SMD: Std mean difference; TKA/THA: Total knee/hip arthroplasty; VAS: Visual analog scale

\section{Acknowledgements}

The research was supported by the Guangxi Medical University First Affiliated Hospital.

\section{Authors' contributions}

JM designed the study. DH and CX developed the inclusion and exclusion criteria. JM conducted the statistical analysis. JM wrote the article. BZ reviewed and approved the article. All authors read and approved the final manuscript.

\section{Funding}

None

\section{Availability of data and materials}

Not applicable

Ethics approval and consent to participate Not applicable

\section{Consent for publication}

Not applicable

\section{Competing interests}

The authors declare that they have no competing interests. 


\section{Author details}

'Department of Bone and Joint Surgery, The First Affiliated Hospital of Guangxi Medical University, Nanning, Guangxi, China. ${ }^{2}$ Guangxi Medical University, Nanning, Guangxi, China.

Received: 24 October 2019 Accepted: 22 January 2020

Published online: 05 February 2020

\section{References}

1. Tilbury C, Haanstra TM, Leichtenberg CS, Verdegaal SH, Ostelo RW, de Vet $\mathrm{HC}$, et al. Unfulfilled expectations after total hip and knee arthroplasty surgery: there is a need for better preoperative patient information and education. J Arthroplasty. 2016;31:2139-45.

2. Koh JC, Song Y, Kim SY, Park S, Ko SH, Han DW. Postoperative pain and patient-controlled epidural analgesia-related adverse effects in young and elderly patients: a retrospective analysis of 2,435 patients. J Pain Res. 2017; 10:897-904.

3. Behman R, Karanicolas PJ, Lemke M, Hanna SS, Coburn NG, Law CH, et al. The effect of early postoperative non-steroidal anti-inflammatory drugs on pancreatic fistula following pancreaticoduodenectomy. J Gastrointest Surg 2015;19:1-8.

4. Dabhi JK, Solanki JK, Mehta A. Antiatherosclerotic activity of ibuprofen, a nonselective COX inhibitor-an animal study. Indian J Exp Biol. 2008;46:476-81.

5. Zidar N, Odar K, Glavač D, Jerše M, Zupanc T, Štajer D. Cyclooxygenase in normal human tissues - is COX-1 really a constitutive isoform, and COX-2 an inducible isoform? J Cell Mol Med. 2010;13:3753-63.

6. Li VG, Seta F, Schwartzman ML, Nasjletti A, Abraham NG. Heme oxygenase attenuates angiotensin II-mediated increase in cyclooxygenase-2 activity in human femoral endothelial cells. Hypertension. 2003;41:715

7. Kaufmann WE, Worley PF, Pegg J, Bremer M, Isakson P. COX-2, a synaptically induced enzyme, is expressed by excitatory neurons at postsynaptic sites in rat cerebral cortex. Proc Natl Acad Sci U S A. 1996;93:2317-21.

8. Minghetti L. Cyclooxygenase-2 (COX-2) in inflammatory and degenerative brain diseases. J Neuropathol Exp Neurol. 2004;63:901-10.

9. Cardwell M, Siviter G, Smith A. Non-steroidal anti-inflammatory drugs and perioperative bleeding in paediatric tonsillectomy. Evid Based Child Health: Cochrane Rev J. 2012;7:244-87.

10. Eren OT, Armağan R, Talmaç MA. Nonsteroidal anti-inflammatory drugs (NSAIDs) and corticosteroids; 2016.

11. Grundmann U, Schreiber JU. Selective cyclooxygenase-2 inhibitors for postoperative pain therapy. Analgesic efficacy and adverse effects. Anaesthesist. 2004;53:1211-8.

12. Asokumar B, Kroin JS, Tuman KJ, Lubenow TR, Dalia E, Mario M, et al. Effects of perioperative administration of a selective cyclooxygenase 2 inhibitor on pain management and recovery of function after knee replacement: a randomized controlled trial. Jama. 2003:290:2411.

13. Rasmussen GL, Malmstrom K, Bourne MH, Jove M, Rhondeau SM, Kotey P, et al. Etoricoxib provides analgesic efficacy to patients after knee or hip replacement surgery: a randomized, double-blind, placebo-controlled study. Anesth Analg. 2005:101:1104-11 table of contents.

14. Viscusi ER, Gimbel JS, Halder AM, Snabes M, Brown MT, Verburg KM. A multiple-day regimen of parecoxib sodium $20 \mathrm{mg}$ twice daily provides pain relief after total hip arthroplasty. Anesth Analg. 2008;107:652-60.

15. Wichai I, Naruemol P, Chutima K, Theerayut I. The post-operative analgesic efficacy of celecoxib compared with placebo and parecoxib after total hip or knee arthroplasty. J Med Assoc Thai. 2010;93:937-42.

16. Li CX. Multimodal analgesia methods for postoperative pain management after total knee arthroplasty. Acta Med Univ Sci Technol Huazhong. 2010;39: 793-6.

17. Ye P, Kou BL, Zhang B. Application of celecoxib in multi-mode analgesia after hip and knee replacement (In Chinese). Chin J Clinicians. 2011;05:153-5.

18. Zhou HM. Efficacy of intravenous parecoxib combined with femoral nerve block for postoperative analgesia and knee functional rehabilitation in elderly patients following total knee replacement. Chin J Anesthesiol. 2011; 31:929-31.

19. Jiang Y. Observation on analgesic effect of celecoxib after total knee replacement. (In Chinese). Chinise J Pract Med. 2012;39:85-6.

20. Rawal N, Viscusi E, Peloso PM, Minkowitz HS, Chen L, Shah S, et al. Evaluation of etoricoxib in patients undergoing total knee replacement surgery in a double-blind, randomized controlled trial. BMC Musculoskelet Disord. 2013;14:1-14
21. Xu F, Yang YZ, Hao J. Multi-mode analgesia of celecoxib after hip and knee replacement. Chinese J Tissue Eng Res. 2015;19:7065-70.

22. Munteanu AM, Cionac Florescu S, Anastase DM, Stoica CI. Is there any analgesic benefit from preoperative vs. postoperative administration of etoricoxib in total knee arthroplasty under spinal anaesthesia? A randomised double-blind placebo-controlled trial. Eur J Anaesthesiol. 2016; 33:840-5.

23. Ding $Y R$, Wang $B$, Wang $W L$, et al. Effectiveness and safety of selective COX2 inhibitor application in the patients after total knee arthroplasty. Pharm Care Res. 2016;16:339-41.

24. Camu F, Borgeat A, Heylen RJ, Viel EJ, Boye ME, Cheung RY. Parecoxib, propacetamol, and their combination for analgesia after total hip arthroplasty: a randomized non-inferiority trial. Acta Anaesthesiol Scand. 2017:61:99-110.

25. Mu DL, Zhang DZ, Wang DX, Wang G, Li CJ, Meng ZT, et al. Parecoxib supplementation to morphine analgesia decreases incidence of delirium in elderly patients after hip or knee replacement surgery: a randomized controlled trial. Anesth Analg. 2017;124:1992-2000.

26. Wang MS. Effect of preemptive analgesia with parexib on pain after total knee replacement. (In Chinese). Medical Forum. 2017;21:1352-4.

27. Yang TM, Si HB, Wu YG, et al. Sequential treatment with parecoxib and celecoxib after primary total knee arthroplasty. Orthop J China. 2017;417: 577-83.

28. Bian YY, Wang LC, Qian WW, Lin J, Jin J, Peng HM, et al. Role of parecoxib sodium in the multimodal analgesia after total knee arthroplasty: a randomized double-blinded controlled trial. Orthop Surg. 2018:10:321-7.

29. Kazerooni R, Tran MH. Evaluation of celecoxib addition to pain protocol after total hip and knee arthroplasty stratified by opioid tolerance. Clin J Pain. 2015;31:903-8

30. Katz WA. Cyclooxygenase-2-selective inhibitors in the management of acute and perioperative pain. Cleve Clin J Med. 2002;69(Suppl 1):Sl65.

31. Hinz B, Brune K. Specific cyclooxygenase-2 inhibitors. Basis and options of a pharmacotherapeutic concept. Anaesthesist. 2000:49:964-71.

32. Javad P, Miller AG, Kishor G. Multimodal pain management after total joint arthroplasty. J Bone Joint Surg (Am Vol). 2011;93:1075.

33. Ji ZW, Bao NR, Zhao JN, Ni JF. Orthopaedics DO. Effect of preoperative cyclooxygenase-2 inhibitor for postoperative pain in patients after total knee arthroplasty:a meta analysis. China J Orthop Traumatol. 2015;28:838.

34. Zhu XT, Chen L, Lin JH. Selective COX-2 inhibitor versus non-selective COX2 inhibitor for the prevention of heterotopic ossification after total hip arthroplasty: a meta-analysis. Medicine. 2018;97:e11649.

35. Bu-Guo XU, Xue DT, Wang XH, Yan SG, Orthopaedics DO. Selective COX-2 inhibitor versus nonselective COX-1 and COX-2 inhibitor in the prevention of heterotopic ossification after total hip arthroplasty: a meta-analysis of randomised trials. Int Orthop. 2011;35:3-8

36. Oh JH, Seo HJ, Lee YH, Choi HY, Joung HY, Kim SH. Do selective COX-2 inhibitors affect pain control and healing after arthroscopic rotator cuff repair? A Preliminary Study. Am J Sports Med. 2017:46:036354651774421.

37. Vastel L, Rosencher $N$, Siney H, J-P C. Prevention of heterotopic ossifications in hip arthroplasty: effectiveness of selective COX-2 inhibitors (celecoxib) versus ketoprofen. Rev Chir Orthop Réparatrice Lappareil Moteur. 2005;91:64

38. Long G, Ji-Yuan D, Zhi-Rui L. Effects of combined application of muscle relaxants and celecoxib administration after total knee arthroplasty (TKA) on early recovery: a randomized, double-blind, controlled study. J Arthroplasty. 2013:28:1301-5.

\section{Publisher's Note}

Springer Nature remains neutral with regard to jurisdictional claims in published maps and institutional affiliations. 